\title{
Combining Microfluidic Secretion Sampling with Small-Volume Proximity Immunoassays: Application to Murine Islets and Adipocytes
}

\author{
Joonyul Kim*, Leah A. Godwin*, Kennon S. Deal*, Zac Keenum*, Desiree Wanders**, Robert L. \\ Judd**, and Christopher J. Easley* \\ * Chemistry and Biochemistry Department, Auburn University, 179 Chemistry Building, Auburn, \\ AL 36849-5312 \\ ** Anatomy, Physiology, and Pharmacology Department, Auburn University, 219 Greene Hall, \\ Auburn, AL 36849-5312
}

The microfluidic platform provides unique opportunities for manipulation and imaging of cellular systems. Various approaches have been developed for culturing, imaging, and sampling from cells in polydimethylsiloxane (PDMS) microdevices [1-3]. However, it is difficult to adapt generallyapplicable immunoassays to the micro- or nanoliter volumes sampled from microfluidic systems. As such, micro-volume assays for specific proteins are typically specialized and lack flexibility for application to other analytes.

Proximity ligation assays (PLA) are among the most sensitive, specific, and flexible protein assays developed to date [4,5]. We have recently developed a modified version of these assays, termed asymmetric proximity ligation assays (aPLA), where assay dynamic range was increased by 100 fold, and sensitivity and limit of detection were also improved [6]. These assays are capable of detecting a wide variety of protein analytes - theoretically any protein with two antibodies-at attomole (or lower) detection limits from only one microliter of sample. We have developed aPLAs for detection of thrombin [6], insulin (FIG. 1a), and adiponectin multimers (FIG. 1b). The dynamic range of the insulin aPLA encompasses the entire range of multiple commercially-available enzymelinked immunosorbent assays (ELISAs), without requiring washing steps. Interestingly, we show that our adiponectin aPLA is more sensitive toward high-molecular-weight multimers of the protein, which have been shown to be the most biologically active forms. These aPLAs are ideal for measurements on microliter-scale secretion samplings from cells in microfluidic systems.

We have also recently developed methods for secretion sampling from eight single pancreatic islets of Langerhans in parallel on a microfluidic device (FIG. 2a). A key advantage offered by our methodology is the passive nature of flow control, where only a single handheld syringe is required to apply vacuum to the outlets of microfluidic channels. Flow rate is passively and accurately controlled by precise patterning of microchannels to specify fluidic flow resistances. Sampling of insulin secretion from many single islets was accomplished, and ELISAs were used to measure insulin. Microfluidic sampling results from single islets were comparable to bulk, tube-based measurements of islets in groups of ten (FIG. 2b), and single-islet volumes could also be acquired using confocal reflectance microscopy (not shown). As shown in previous work [1], it should be possible to modify the microfluidic system for passive sampling into aqueous-in-oil droplets, thereby greatly improving temporal resolution on secretion measurements.

By combining homogeneous, small-volume protein assays such as aPLA with microfluidic sampling, measurements can be expanded to a variety of cytokines or adipokines. For example, insulin or glucagon from islets, as well as adiponectin or leptin secreted from adipocytes. We have 
begun applying our microfluidic systems to murine adipocyte sampling, and we were able to culture primary adipocytes into a 3D collagen matrix in microfluidic reservoirs. Adiponectin secretion was measured from the cells, with overall magnitudes near that of multi-well plate experiments (FIG. 2c). However, with the current microfluidic system, we were unable to distinguish between secretory products of insulin-treated adipocytes from untreated adipocytes. In future studies, we will optimize the microfluidic interface for secretion sampling of adipocytes, providing a unique system capable of studying fundamentals of acute adiponectin secretion. We will also begin to investigate the dependence of insulin secretion from pancreatic islets on the cellular architecture, i.e. the relative cell composition of each islet ( $\%$ alpha cells, $\%$ beta cells, etc.), in relation to paracrine signaling.

\section{References}

[1] C.J. Easley et al., Anal. Chem. 81 (2009) 9086.

[2] R. Gomez-Sjoberg et al., Anal. Chem. 79 (2007) 8557.

[3] C.S. Nunemaker et al., PLoS One 4 (2009) e8428.

[4] S. Fredriksson et al., Nature Biotechnol. 20 (2002) 448.

[5] M. Gullberg et al., Proc. Natl. Acad. Sci. USA 101 (2004) 8420.

[6] J. Kim et al., Anal. Chem. 82 (2010) 6976.

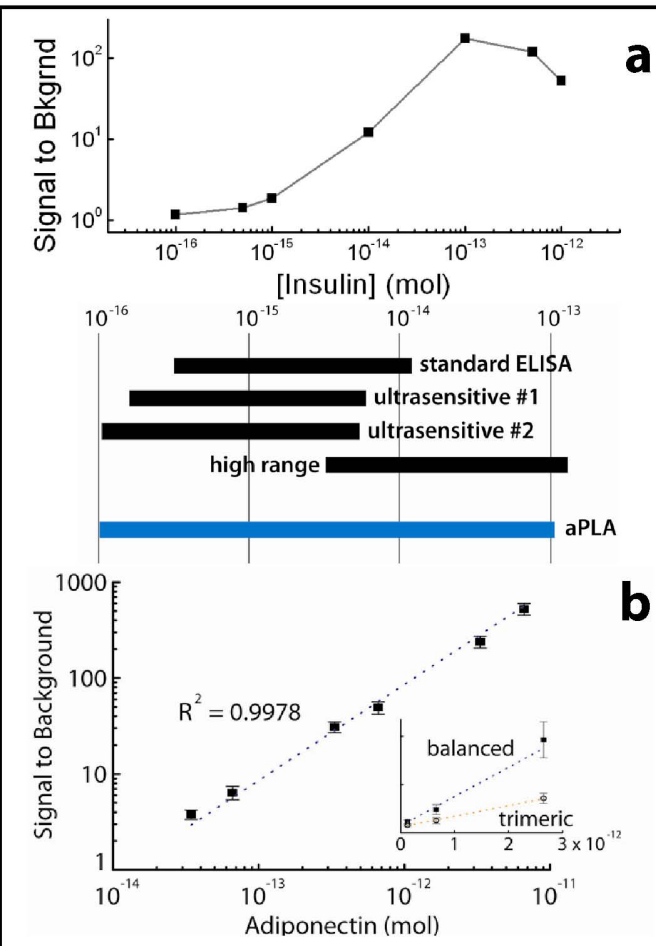

FIG. 1. Antibody-oligo based aPLA has been proven successful with novel (a) insulin and (b) adiponectin aPLAs.

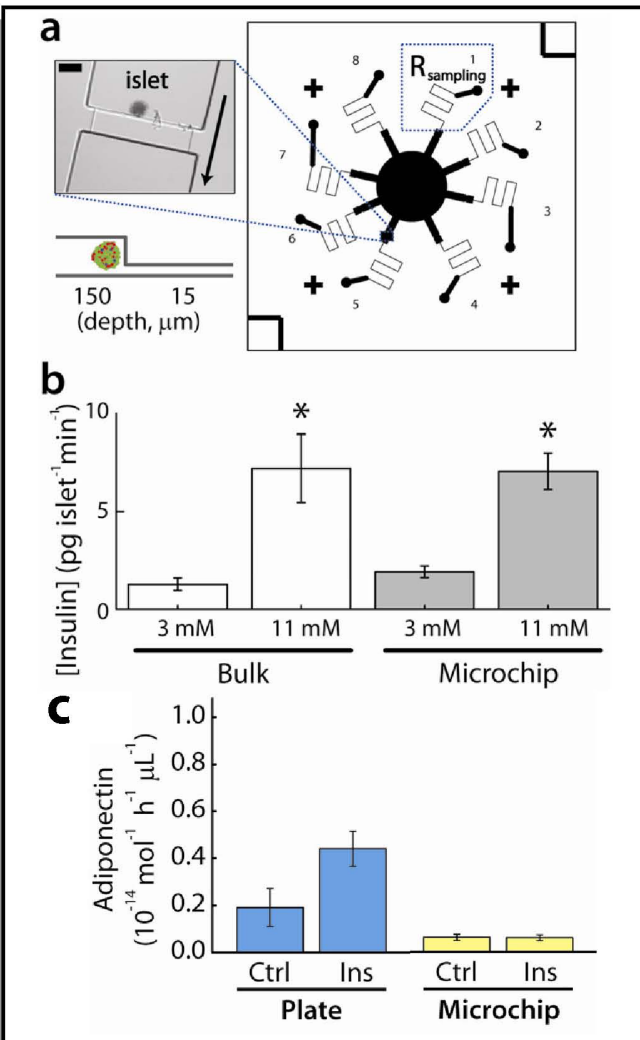

FIG. 2. (a) Passive microfluidic sampling was used for insulin measurements from 8 islets in parallel. (b) Bulk measurements from 380 islets $(5$ mice) correlated well with microfluidic sampling from 66 islets (5 mice). (c) Devices were also modified for secretion sampling from primary murine adipocytes. $($ scale bar $=100 \mu \mathrm{m})$ 